\title{
BIVARIANT LONG EXACT SEQUENCES II
}

\author{
T.H. FAY AND K.A. HARDiE
}

\begin{abstract}
Given a pair of short exact sequences

1) $0 \rightarrow X \stackrel{\gamma}{\rightarrow} Y \stackrel{6}{\rightarrow} Z \rightarrow 0,0 \rightarrow A \stackrel{a}{\rightarrow} B \stackrel{\beta}{\rightarrow} C \rightarrow 0$

in an abelian category $A_{\sim}$, with sufficiently many projectives and injec-

tives, and given an additive bifunctor $T$ we show that $T$ applied to the pair (1) gives rise to a diagran of a type described by C. T. C. Wall that contains 15 interlocking long exact sequences involving the derived functors of $T$ at $(A, X),(A, Y)$, etc. and also involving the derived functors of $T_{P}$ and $T_{Q}$ which are two functors with domain $A^{2}$ that arise through the failure of $T$ to preserve pullbacks and pushouts. In the case of Hom (respectively $\otimes$ ) in the category of $G$-modules for a group $G$ the derived functors of $T_{P}$ (respectively $T_{Q}$ ) are expressed in terms of group cohomology (respectively homology)
\end{abstract}

\section{0 . Introduction}

Given two exact sequences

$$
0 \rightarrow X \stackrel{\gamma}{\rightarrow} Y \stackrel{\delta}{\rightarrow} Z \rightarrow 0,0 \rightarrow A \stackrel{\alpha}{\rightarrow} B \stackrel{\beta}{\rightarrow} C \rightarrow 0
$$

in an abelian category $\underset{\sim}{A}$, Pressman [7] showed that there is a long exact "bivariant" sequence

$$
0 \rightarrow \operatorname{Hom}(Z, A) \rightarrow \operatorname{Hom}(Y, B) \rightarrow \operatorname{hom}(\gamma, \beta) \rightarrow \operatorname{Ext}^{1}(Z, A) \rightarrow
$$

$$
\rightarrow \operatorname{Ext}^{1}(Y, B) \rightarrow \operatorname{ext}^{1}(\gamma, \beta) \rightarrow \ldots
$$


that generalizes the usual univariant Hom-Ext sequences associated with the pair (0.1) and interlocks with them:

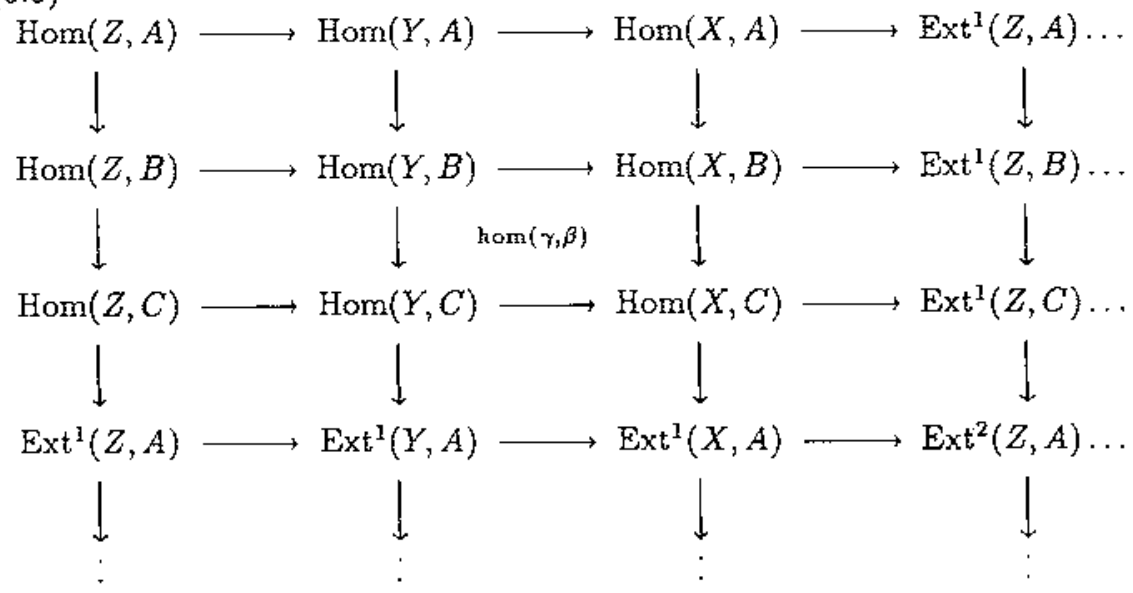

In the above diagram, Pressman's arrows are shown dotted. The triangles are commutative and, as is well known, the rectangles of the horizontal and vertical array are all commutative except that those involving four coboundary arrows are anticommutative. In the sequence $(0.2)$ the functors hom $(-,-)$ and ext ${ }^{1}$ $(-,-)$ etc., refer to the hom and extension functors defined in the category ${\underset{\sim}{\sim}}^{2}$ (of arrows of $\underset{\sim}{A}$ ), known also to be abelian.

In attempting to obtain an analogue of Pressman's sequence for an axbitrary additive bifunctor $T$, Hardie [6] observes that the definition of hom in $\underset{\sim}{A^{2}}$ sets up a pullback diagram

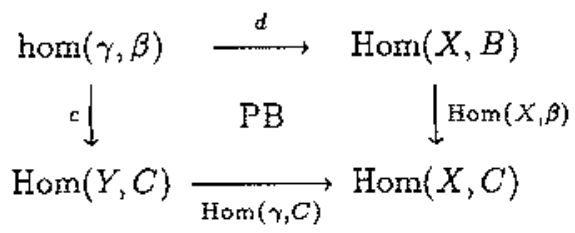

where $d$ and $c$ denote domain and codomain restriction operators respectively. Accordingly, given a bifunctor $T$, covariant in the first variable and contravariant in the second, he applies $T$ to the (evidently) bicartesian square

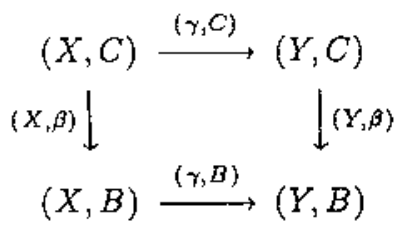


in $\underset{\sim}{A} \times{\underset{\sim}{A}}^{o p}$ to obtain the (outer) rectangle

$$
\begin{gathered}
T(X, C) \underset{T_{P}(\gamma, \beta)}{\stackrel{T(\gamma, C)}{\longrightarrow}} T(Y, C) \\
\left.T(X, \beta)\right|_{\downarrow} T(Y, B) \\
T(X, B) \underset{T(\gamma, B)}{\rightarrow} T(Y, B)
\end{gathered}
$$

and then defines $T_{P}(\gamma, \beta)$ (respectively $T_{Q}(\gamma, \beta)$ ) by requiring that it complete a pullback (respectively pushout) rectangle with the relevant horizontal and vertical arrows. In this way $T_{P}$ and $T_{Q}$ become bifunctors with the same variance as $T$ that measure the failure of $T$ to preserve pullbacks and pushouts. It is clear how to modify the definition for functors of other variance and, in particular, we recover hom $(\gamma, \beta)=\operatorname{Hom}_{p}(\gamma, \beta)$.

From henceforth assume that $\underset{\sim}{A}$ has sufficient injectives and projectives. Then using the terminology of Cartan and Eilenberg [3, p. 82], recall that $R^{n} T$, the n'th right derived functor of $T$ evaluated at $(A, B)$, is the n'th component of the graded object

$$
R T(A, B)=H\left(T\left(\underset{\sim}{E_{A}} \underset{\sim}{P} B\right)\right),
$$

the (co)-homology of the right complex over $T(A, B)$ that $T$ associates with the injective resolution $\underset{\sim}{E_{A}}$ of $A$ and the projective resolution $\underset{\sim}{P_{B}}$ of $B$. If one applies this machinery to the pair of short exact sequences $(0.1)$ as is done $[3 ; \mathrm{V}$, Proposition 4] one obtains a doubly infinite diagram (called in [6] a cylinder-web diagram) combining the long exact sequences of $R T$ at $(A, X)$, $(A, Y),(A, Z),(B, X),(B, Y),(B, Z),(C, X),(C, Y)$ and $(C, Z)$. It was shown in $[6$, Theorem 1$]$ that three "diagonal" sequences

$$
\begin{aligned}
& 0 \rightarrow R^{0} T(A, Z) \rightarrow R^{0} T(B, Y) \rightarrow R^{0} T_{P}(\beta, \gamma) \rightarrow R^{1} T(A, Z) \rightarrow \ldots \\
& 0 \rightarrow R^{0} T(A, X) \rightarrow R^{0} T_{P}(\beta, \gamma) \rightarrow R^{0} T(C, Y) \rightarrow R^{1} T(A, X) \rightarrow \ldots \\
& 0 \rightarrow R^{0} T(C, Z) \rightarrow R^{0} T_{P}(\beta, \gamma) \rightarrow R^{0} T(B, X) \rightarrow R^{1} T(C, Z) \rightarrow \ldots
\end{aligned}
$$

each passing through the groups $R^{n} T_{P}(\beta, \gamma)(n \geq 0)$, interlock with the sequences of the diagram. The sequence 0.4 can be regarded as the desired analogue of Pressman's sequence.

There are indications, however, that the above is not the full story. Firstly, using the original technique of Pressman, we have derived six further diagonal sequences that interlock with 0.3 [4]. Secondly, we obtained in [4] canonical isomorphisms

$$
\operatorname{ext}^{n}(\gamma, \alpha) \approx \operatorname{ext}^{n}(\delta, \beta)
$$


Thirdly, Ross Street [11] has shown that if one applies the homology functor to a $3 \times 3$ diagram of chain complexes, then one obtains a diagram of a type considered by Wall [12] and described by Street [11] as a three-diamond diagram. We prove the following theorem.

0.8 Theorem. Given an additive bifunctor $T$ covariant in the first variable, contravariant in the second, and given a pair of short exact sequences 0.1 , there exist:

(i) in addition to the sequences $0.4,0.5,0.6$ the exact sequences

$$
\begin{aligned}
& 0 \rightarrow R^{0} T(B, Z) \rightarrow R^{0} T_{P}(\beta, \delta) \rightarrow R^{0} T(A, X) \rightarrow R^{1} T(B, Z) \rightarrow \ldots \\
& 0 \rightarrow R^{0} T_{P}(\beta, \delta) \rightarrow R^{0} T(B, Y) \rightarrow R^{0} T(C, X) \rightarrow R^{1} T_{P}(\beta, \delta) \rightarrow \ldots
\end{aligned}
$$$$
0 \rightarrow R^{0} T(A, Y) \rightarrow R^{0} T_{P}(\beta, \delta) \rightarrow R^{0} T(C, Z) \rightarrow R^{1} T(A, Y) \rightarrow \ldots
$$

$$
0 \rightarrow R^{0} T_{P}(\alpha, \delta) \rightarrow R^{0} T(A, Y) \rightarrow R^{0} T(B, X) \rightarrow R^{1} T_{P}(\alpha, \delta) \rightarrow \ldots
$$

$$
0 \rightarrow R^{0} T_{P}(\alpha, \delta) \rightarrow R^{0} T(B, Z) \rightarrow R^{0} T(C, Y) \rightarrow R^{1} T_{P}(\alpha, \delta) \rightarrow \ldots
$$

$$
\begin{aligned}
0 \rightarrow R^{0} T(A, Z) & \rightarrow R^{0} T_{P}(\alpha, \delta) \rightarrow 0 \rightarrow R^{1} T(A, Z) \rightarrow R^{1} T_{P}(\alpha, \delta) \\
& \rightarrow R^{0} T(C, X) \rightarrow \ldots
\end{aligned}
$$

(ii) the following natural isomorphisms

$$
\begin{aligned}
& R^{n} T_{P}(\alpha, \gamma) \approx R^{n} T_{P}(\beta, \delta) \approx R^{n} T_{Q}(\alpha, \delta) \\
& R^{n} T_{P}(\beta, \gamma) \approx R^{n} T_{Q}(\alpha, \gamma) \approx R^{n} T_{Q}(\beta, \delta) .
\end{aligned}
$$

0.17. Remark. The sequences described form together with the cylinderweb diagram a diagram of the type described by Wall [12].

0.18 . Remark. Information relating $R^{n} T_{Q}(\beta, \gamma)$ appears to be missing from the above. In fact there is also a natural isomorphism

$$
R^{n} T_{P}(\alpha, \delta) \approx R^{n-1} T_{Q}(\beta, \gamma)(n>1) .
$$

A proof will be given in a subsequent paper.

A version of the theory can also be given for left derived functors. In section 4 we display the Wall diagrams generated by Hom and $\otimes$. What is the role of the additional exact sequences? In the first place as pointed out by Street [11], there is reason to believe that the full Wall diagram contains information inaccessibie to the standard horizontal and vertical sequences of the cylinder-web 
diagram. In homotopy pair theory [8], [9] corresponding diagonal sequences arise that pass through the so-called homotopy pair groups. These additional objects appear to be the natural home for secondary homotopy compositions (Toda brackets) in the sense that the brackets live there with zero indeterminacy. Similar considerations apply (for example) to secondary Yoneda products in the hom-ext Wall diagram. In a subsequent paper the authors hope to show how the additional objects $R^{n} T_{P}(\beta, \delta)$ et al can be computed and the sequence structure exploited. Preliminary results in this direction are given in section 5 for the cases of hom and $\otimes$ in the category of $G$-modules for a group $G$.

\section{Preliminary Lemmas}

We first recall the $3 \times 3$ diagonal lemma $[6, \mathrm{p}, 169]$. Suppose that in the following diagram in an abelian category the sequences

$$
\cdot \stackrel{a_{i}}{\longrightarrow} \cdot \stackrel{b_{i}}{\longrightarrow} \cdot, \stackrel{x_{i}}{\longrightarrow} \cdot \stackrel{y_{i}}{\longrightarrow} \cdot
$$

make up a commutative array of short exact sequences and suppose that $b^{\prime}$ and $y^{\prime}$ complete the pullback of $b_{3}$ and $y_{3}$.

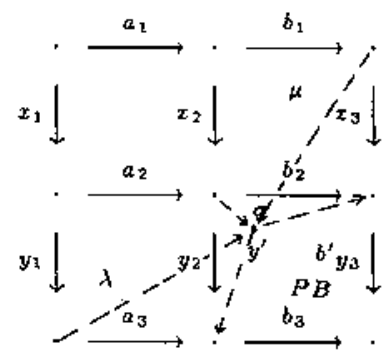

Then there exist unique morphisms $\sigma, \lambda$ and $\mu$ such that (a) $y^{\prime} \sigma=y_{2}$ and $b^{\prime} \sigma=b_{2}$, (b) $y^{\prime} \lambda=a_{3}$ and $b^{\prime} \lambda=0$, (c) $y^{\prime} \mu=0$ and $b^{\prime} \mu=x_{3}$. Let $\rho=x_{2} a_{1}=$ $a_{2} x_{1}$.

1.1. $\left(3 \times 3\right.$ Diagonal) Lemma. The sequences $\cdot \stackrel{\rho}{\rightarrow} \cdot \stackrel{\sigma}{\rightarrow} \cdot, \cdot \stackrel{\lambda}{\rightarrow} \cdot \stackrel{b^{\prime}}{\rightarrow} \cdot$ and $\cdot \stackrel{\mu}{\rightarrow} \cdot \stackrel{y^{\prime}}{\rightarrow}$. are short exact.

The scope of the $3 \times 3$ diagonal lemma is considerably expanded by the following two square lemma. Suppose that in the following commutative diagram in an abelian category, the horizontal rows are short exact sequences; let $z^{\prime}$ and $d^{\prime}$ complete the pullback of $z$ and $d$, let $a^{\prime}$ and $x^{\prime}$ complete the pushout of $a$ and $x$.

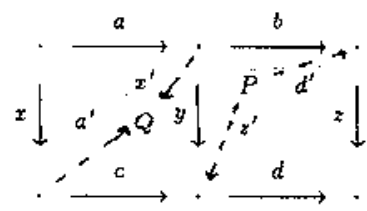


1.2. (Two Square) Lemma. There exists a unique morphism $\lambda: Q \rightarrow P$ satisfying $z^{\prime} \lambda a^{\prime}=c, z^{\prime} \lambda x^{\prime}=y, d^{\prime} \lambda x^{\prime}=b$ and $d^{\prime} \lambda a^{\prime}=0$. Furthermore $\lambda$ is on isomorphism.

A proof of the Two Square Lemma can be found in [5]. We shall require also the following lemma which may perhaps be a known result.

1.5 Lemma. Let $A \stackrel{\varphi}{\rightarrow} B \stackrel{\uplus}{\rightarrow} C$ and $K \stackrel{\theta}{\rightarrow} A^{\prime} \stackrel{\varphi^{\prime}}{\rightarrow} B^{\prime}$ be short exact sequences of cochain complexes and let

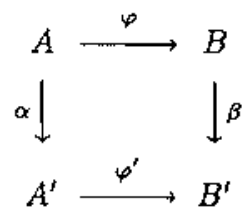

be a commuting square of cochain maps. Then there is a homomorphism $\Delta$ : $H^{n}(C) \rightarrow H^{n+1}(K)$ completing the lodder diagram

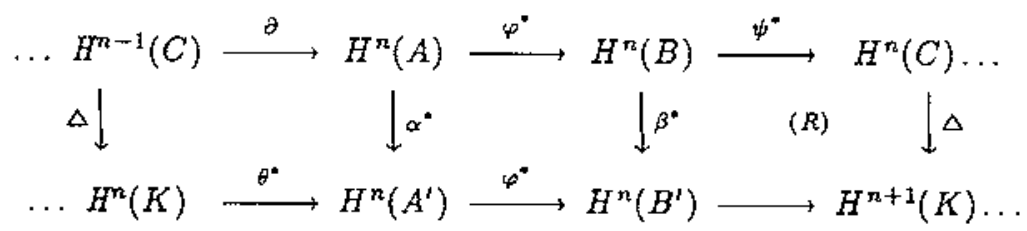

in which the horizontal rows are the associated long cohomology sequences and the rectangle $(R)$ is anticommutative.

Proof: Let $c \in C^{n}$ be such that $d c=0 \in C^{n+1}$. Then there exists $a \in A^{n+1}$, $b \in B^{n}$ such that $\psi b=c, \phi a=d b$ and $d a=0$. Moreover there exists an $a^{\prime}$ such that $\varphi^{\prime} a^{\prime}=\beta b$. Then $\varphi^{\prime}\left(\alpha a-d a^{\prime}\right)=0$. Hence there exists a $k \in K^{n+1}$ such that $\theta k=\alpha a-d a^{\prime}$. Then $d k=0$ and $\Delta c l s(c)=c l s(k)$ is independent of choices. We may check readily that $(R)$ is anticommutative and also the commutativity of the remaining rectangles.

1.6. Remark. If, in Lemma 1.5 , it is known that $\alpha^{*}$ and $\beta^{*}$ are isomorphisms, then of course we can infer that $\Delta$ is an isomorphism in each dimension.

\section{Resolutions in $\underset{\sim}{A^{2}}$}

Since $\underset{\sim}{A}$ has sufficient injectives and projectives, by a standard technique we may associate with the short exact sequences 0.1 a short exact sequence of injective resolutions

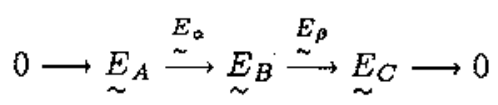


and a short exact sequence of projective resolutions

$$
0 \longrightarrow P_{\sim}^{X} \stackrel{p^{\gamma}}{\longrightarrow} P_{\sim}^{Y} \stackrel{P^{\delta}}{\longrightarrow} P_{\sim}^{Z} \longrightarrow 0 .
$$

Now the projective (respectively injective) objects in $A_{\sim}^{2}$ are precisely the coretractions $P_{1} \rightarrow P_{2}$ between projectives in $A$ (respectively the retractions $E_{1} \rightarrow E_{2}$ between injectives in $\left.\underset{\sim}{A}\right)[7$, Proof of Lemma 5.3]. Hence we can regard the cochain map $\underset{\sim}{E_{\beta}}$ as an injective resolution of $\beta$ and the chain map $\underset{\sim}{P^{\gamma}}$ as a projective resolution of $\gamma$. By definition of the derived functors we have the equations

$$
\begin{aligned}
& R^{n} T_{P}(\beta, \gamma)=H^{n}\left(T_{P}\left(\underset{\sim}{E_{\beta}}, \underset{\sim}{P^{\gamma}}\right)\right) \\
& R^{n} T_{Q}(\beta, \gamma)=H^{n}\left(T_{Q}\left(\underset{\sim}{E_{\beta}} \underset{\sim}{P^{\gamma}}\right)\right) .
\end{aligned}
$$

Since the component arrows of $\underset{\sim}{E_{\alpha}}$ are not retractions, $\underset{\sim}{E_{\alpha}}$ is not an injective resolution of $\alpha$. However it is easy to see that $\underset{\sim}{E_{\alpha}}$ (respectively $P_{\sim}^{\delta}$ ) is an acyclic complex over $\alpha$ (respectively $\delta$ ). These acyclic resolutions can nevertheless be used to compute the derived functors of $T_{P}$ and $T_{Q}$ :

2.3. Proposition
(a) $R^{n} T_{P}(\alpha, \gamma) \approx H^{n}\left(T_{P}\left(\underset{\sim}{E_{\alpha}}, \underset{\sim}{P^{\gamma}}\right)\right)$
(b) $R^{n} T_{P}(\beta, \delta) \approx H^{n}\left(T_{P}\left(\underset{\sim}{E}{ }_{\beta}, \underset{\sim}{P^{\delta}}\right)\right)$
(c) $R^{n} T_{Q}(\alpha, \delta) \approx H^{n}\left(T_{Q}\left(\underset{\sim}{E_{\alpha}}, P_{\sim}^{\delta}\right)\right)$
(d) $R^{n} T_{Q}(\alpha, \gamma) \approx H^{n}\left(T_{Q}\left(\underset{\sim}{E_{\alpha}}, P_{\sim}^{\gamma}\right)\right)$
(e) $R^{n} T_{Q}(\beta, \delta) \approx H^{n}\left(T_{Q}\left(\underset{\sim}{E_{\beta}}, P_{\sim}^{\delta}\right)\right)$.

Proof: (a) In the diagram

$$
T\left({\underset{\sim}{E}{ }_{B}, P^{Z}}^{Z}\right) \longrightarrow T\left(\underset{\sim}{E_{B}, P_{\sim}^{P}}\right) \rightarrow T\left(\underset{\sim}{E_{B}, \sim_{\sim}^{P}}\right)
$$

the horizontal row is the short exact sequence of cochain complexes obtained by applying $T\left(\underset{\sim}{E_{B}},--\right)$ to 2.2 and the right hand vertical arrow is induced by the chain map $\underset{\sim}{E_{\alpha}}$. As discussed in $[6, p .174]$, the rectangle is a pullback and hence the diagonal sequence is also short exact. Since $A^{2}$ has sufficient injectives there 
exists an injective resolution $\underset{\sim}{F_{\alpha}}: \underset{\sim}{F_{A}} \rightarrow \underset{\sim}{F_{B}}$ of $\alpha$ and a morphism of resolutions $\underset{\sim}{E_{\alpha}} \rightarrow \underset{\sim}{F_{\alpha}}$. It follows that there is a diagram like 2.4 in which $\underset{\sim}{F_{\alpha}}$ replaces $\underset{\sim}{E_{\alpha}}$ and hence also a morphism of the diagonal short exact sequences:

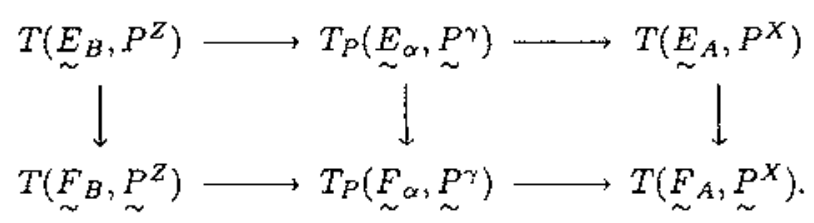

Taking cohomology the resulting morphism of long exact sequences induces isomorphisms, via the 5-Lemma, so that we have:

$$
H^{n}\left(T_{P}\left(\underset{\sim}{E}, \underset{\sim}{E^{\gamma}}\right)\right) \approx H^{n}\left(T_{P}\left(\underset{\sim}{F}, \underset{\sim}{P^{\gamma}}\right)\right) \approx R^{n} T_{P}(\alpha, \gamma) .
$$

(b) The argument is similar to part (a).

(c) In the diagram

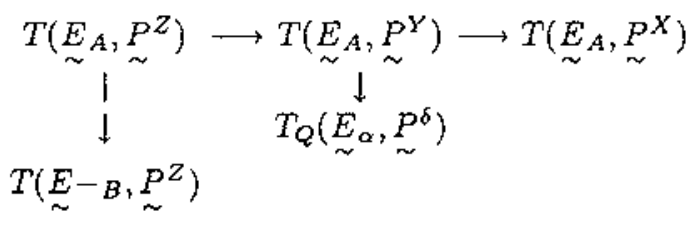

the horizontal sequence is short exact obtained by applying $T\left(\underset{\sim}{E_{A}},--\right)$ to 2.2 and the left hand vertical arrow is induced by $\underset{\sim}{E_{\alpha}}$. The rectangle is a pushout and the diagonal sequence is short exact. Since $A^{2}$ has sufficient projectives, there exists a projective resolution $\underset{\sim}{Q^{\delta}}: \underset{\sim}{Q^{Y}} \rightarrow \underset{\sim}{Q^{\widetilde{z}}}$ and diagrams similar to 2.5 with $\underset{\sim}{F_{A}}$ replacing $\underset{\sim}{E_{A}}$ (respectively $\underset{\sim}{F_{A}}$ replacing $\underset{\sim}{E_{A}}$ and $\underset{\sim}{Q^{\delta}}$ replacing $\underset{\sim}{P^{\delta}}$ ). The corresponding diagonal sequences are linked by morphisms:

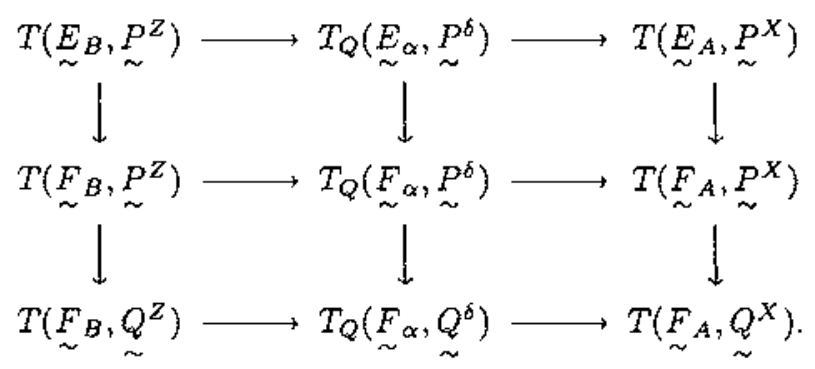

Taking cohomology and applying the 5-Lemma argument we obtain natural isomorphisms

$$
H^{n}\left(T_{Q}\left(\underset{\sim}{E}, \underset{\sim}{P^{\delta}}\right)\right) \approx H^{n}\left(T_{Q}\left(\underset{\sim}{F_{\alpha}}{\underset{\sim}{Q^{\delta}}}_{\sim}\right)\right) \approx R^{n} T_{Q}(\alpha, \delta) .
$$


Similar arguments dispose of parts (d) and (e), completing the proof of Proposition 2.3 .

We may also complete the proof of part (ii) of Theorem 0.8 by constructing appropriate pullbacks and pushouts in the following $3 \times 3$ diagram (obtained by applying $T(-,-,-)$ to 2.1 and 2.2$)$ and making various applications of the Two-Square Lemma.

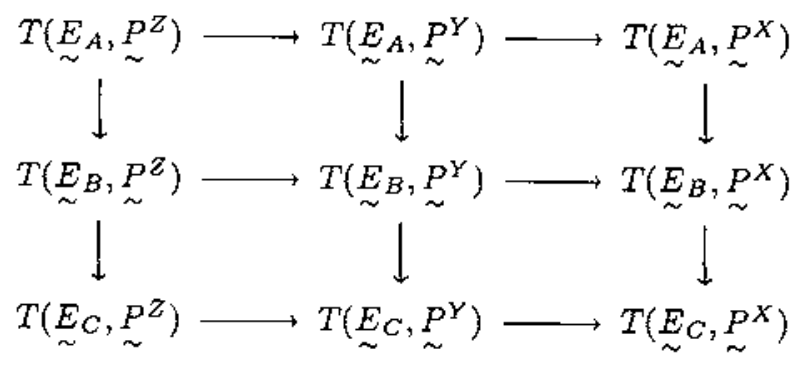

\section{The Long Diagonal Sequences}

The sequence 0.9 of theorem 0.8 is obtained by applying cohomology to the diagonal short exact sequence in 2.4 and recalling the isomorphism 0.15 . The sequence 0.10 is obtained by constructing the pushout complex $T_{Q}\left(\underset{\sim}{E_{\alpha}},{\underset{\sim}{p}}^{\delta}\right)$ : the dual of the $3 \times 3$ Diagonal Lemma yields the short exact sequences:

$$
\begin{aligned}
& T_{Q}\left(\underset{\sim}{E_{\alpha}, P_{\sim}}\right) \rightarrow T\left(\underset{\sim}{E_{B}}, \underset{\sim}{P^{Y}}\right) \rightarrow T\left(\underset{\sim}{E_{C}}, \underset{\sim}{P^{X}}\right) \\
& T\left(\underset{\sim}{E_{A}}, \underset{\sim}{P^{Y}}\right) \rightarrow T_{Q}\left(\underset{\sim}{E_{\alpha}},{\underset{\sim}{P}}^{\delta}\right) \rightarrow T\left(\underset{\sim}{E_{C}},{\underset{\sim}{P}}^{Z}\right) .
\end{aligned}
$$

The first of these gives rise to 0.10 and the second to 0.11 . To complete the proof of Theorem 0.8 requires an analysis of the resolutions $\underset{\sim}{F}$ and $\underset{\sim}{Q^{\delta}}$. Suppose that

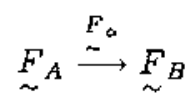

is an injective resolution of $\alpha$, where $\underset{\sim}{F_{A}}$ is the injective resolution

$$
0 \rightarrow A \stackrel{i_{A}}{\rightarrow} F_{A}^{0} \rightarrow F_{A}^{1} \rightarrow F_{A}^{2} \rightarrow \ldots
$$

regarded as a right complex over $A[3$, p. 78$]$. Then $\Sigma A=\operatorname{coker} i_{A}$ and $\Sigma B=$ coker $i_{B}$ are suspensions of $A$ and $B$ and the induced arrow $\Sigma \alpha: \Sigma A \rightarrow \Sigma B$ is epic since the $F_{\alpha}^{i}: F_{A}^{i} \rightarrow F_{B}^{i}$ are retractions. Then $E^{i}=$ ker $F_{\alpha}^{i}$ is an injective 
$(i \geq 0)$ and we have the following diagram in which $K=\operatorname{ker} \Sigma \alpha$.

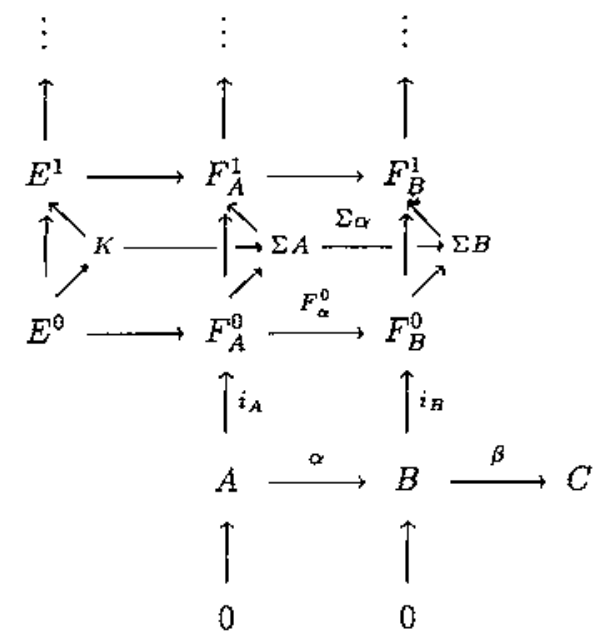

By an application of the Ker-Cok-Lemma $[1,10.3 .1]$ we find that

$$
0 \rightarrow E^{0} \rightarrow K \rightarrow C \rightarrow 0
$$

is exact. Moreover, since $E^{0}$ is injective the sequence splits. Since $E^{0} \rightarrow E^{1}$ also splits, there exists an injective object $E$ such that $E^{1} \approx E^{0} \oplus E$ and a commutative diagram

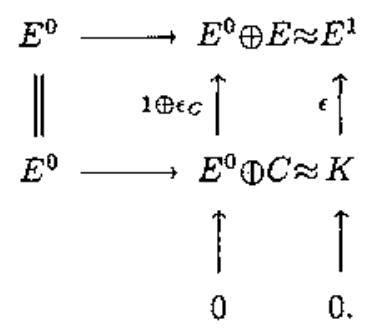

Consider now the right complexes

$$
\begin{aligned}
& \underset{\sim}{E}=\left(E^{0} \rightarrow E^{1} \rightarrow E^{2} \rightarrow \ldots\right) \\
& \underset{E^{\prime}}{\sim}=\left(E^{0} \rightarrow E^{0} \rightarrow 0 \rightarrow \ldots\right) \\
& \underset{\sim}{E^{\prime \prime}}=\left(E \rightarrow E^{2} \rightarrow E^{3} \rightarrow \ldots\right)
\end{aligned}
$$

and note that the monomorphism $C \stackrel{\epsilon}{\rightarrow} E$ enables us to regard $E^{\prime \prime}$ as an injective resolution of $C$. Recalling that the suspension $\Sigma \underset{\sim}{X}$ of a complex $\underset{\sim}{X}$ is such that $(\Sigma \underset{\sim}{X})^{n}=\underset{\sim}{X^{n-1}}$, we have the following lemma. 
3.4. Lemma. $\underset{\sim}{E} \approx \underset{\sim}{E^{i}} \oplus \Sigma \underset{\sim}{E^{\prime \prime}}$

3.5. Remark. In the short exact sequence $K \rightarrow \Sigma A \stackrel{\Sigma \alpha}{\rightarrow} \Sigma B$, the module $K$ has the same Eckmann-Hilton injective homotopy type as $C$. Dually, let

$$
Q_{\sim}^{Y} \stackrel{Q^{s}}{\longrightarrow} Q_{\sim}^{Z}
$$

be a projective resolution of $\delta$, where $Q^{Z}$ is the projective resolution

$$
\cdots \rightarrow Q_{2}^{Z} \rightarrow Q_{1}^{Z} \rightarrow Q_{0}^{Z} \stackrel{\epsilon_{A}}{\rightarrow} Z \rightarrow 0
$$

regarded as a left complex over $Z$. There is a diagram dual to 3.2 in which $\Omega Y=\operatorname{ker} \epsilon_{Y}$ and $\Omega Z=$ ker $\epsilon_{Z}$ are loop spaces of $Y$ and $Z$; the induced arrow $\Omega \delta: \Omega Y \rightarrow \Omega Z$ is monic, since $Q_{i}^{\delta}: Q_{i}^{Y} \rightarrow Q_{i}^{Z}$ are coretractions; $P_{i}=\operatorname{coker} Q_{i}^{\delta}$ is a projective $(i \geq 0) ; J=\operatorname{coker} \Omega \delta$ :

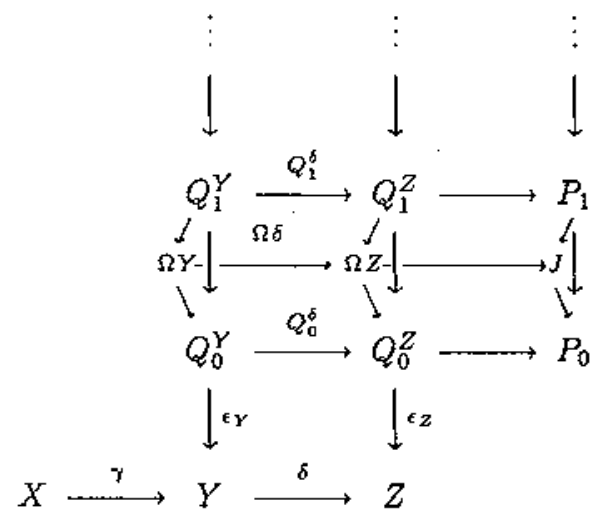

By another application of the Ker-Cok Lemma we have

$$
0 \rightarrow X \rightarrow J \rightarrow P_{0} \rightarrow 0
$$

is exact. Moreover, since $P_{0}$ is projẹctive, the sequence splits. Since $P_{1} \rightarrow P_{2}$ also splits, there exists a projective object $P$ such that $P_{1} \approx P_{0} \oplus P$. By a dual of Lemma 3.4 we have

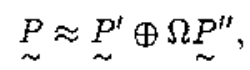

where the left complexes $\underset{\sim}{P}, \underset{\sim}{P^{\prime}}, \underset{\sim}{P^{\prime \prime}}$ are as indicated and $\left.\underset{\sim}{(\Omega X}\right)_{n}=\underset{\sim}{X}{ }_{n-1}$ :

$$
\begin{aligned}
& \underset{\sim}{P}=\left(\cdots \rightarrow P_{2} \rightarrow P_{1} \rightarrow P_{0}\right) \\
& \stackrel{P}{ }^{\prime}=\left(\cdots \rightarrow 0 \rightarrow P_{0} \rightarrow P_{0}\right) \\
& \underset{\sim}{P^{\prime \prime}}=\left(\cdots \rightarrow P_{3} \rightarrow P_{2} \rightarrow P\right) .
\end{aligned}
$$


The epimorphism $P \rightarrow X$ provides $P_{\sim}^{\prime \prime}$ with the structure of a projective resolution of $X$.

3.10. Remark. In the short exact sequence $\Omega Y \stackrel{\Omega \delta}{\rightarrow} \Omega Z \rightarrow J$, the module $J$ has the Eckmann-Hilton projective homotopy type of $X$.

Now, by applying $T$ to the pair of short exact sequences

$$
\begin{aligned}
& \underset{\sim}{E} \underset{\sim}{\longrightarrow} \underset{\sim}{F_{A}} \stackrel{\stackrel{F_{\alpha}}{\longrightarrow}}{\longrightarrow} F_{B} \\
& Q^{Y} \stackrel{Q^{6}}{\sim} Q^{Z} \rightarrow \underset{\sim}{\sim}
\end{aligned}
$$

we obtain another diagram of type 2.6. Considering the pullback of the arrows

$$
\begin{aligned}
& T\left({\underset{\sim}{F}}_{B},{\underset{\sim}{Q}}^{\delta}\right): T\left(\underset{\sim}{F_{B}},{\underset{\sim}{Q}}^{Z}\right) \rightarrow T\left(\underset{\sim}{F_{B}},{\underset{\sim}{Q}}^{Y}\right) \\
& T\left(\underset{\sim}{F},{\underset{\sim}{Q}}^{Y}\right): T\left(\underset{\sim}{F},{\underset{\sim}{Q}}^{Y}\right) \rightarrow T\left(\underset{\sim}{F},{\underset{\sim}{Q}}^{Y}\right)
\end{aligned}
$$

gives rise to short exact sequences

$$
\begin{aligned}
& T\left(\underset{\sim}{F_{B}}, \underset{\sim}{P}\right) \rightarrow T_{P}\left({\underset{\sim}{\alpha}}_{\alpha},{\underset{\sim}{Q}}^{\delta}\right) \rightarrow T\left(\underset{\sim}{F_{A}},{\underset{\sim}{Q}}^{Y}\right) \\
& T\left(\underset{\sim}{E}, \underset{\sim}{Q^{Y}}\right) \rightarrow T_{P}\left(\underset{\sim}{F},{\underset{\sim}{Q}}^{\delta}\right) \rightarrow T\left(\sim_{\sim}^{F_{B}}, \sim^{Z}\right) \\
& T(\underset{\sim}{E}, \underset{\sim}{P}) \rightarrow T\left(\underset{\sim}{F_{A}},{\underset{\sim}{Q}}^{Z}\right) \rightarrow T_{P}\left(\underset{\sim}{F_{\alpha}},{\underset{\sim}{Q}}^{\delta}\right)
\end{aligned}
$$

whose exact cohomology sequences are interpretable, in view of 3.4 and 3.9 , as the desired sequences $0.12,0.13$ and 0.14 completing the proof of Theorem 0.8 .

3.16. Remark. The compatibility of the diagonal sequences $0.9,0.10,0.11$ with the horizontal and vertical sequences of the cylinder-web diagram is a consequence of the universality properties of the pushout and pullback in the lemmas 1.1 and 1.2. In the case of the sequences $0.12,0.13,0.14 \mathrm{it}$ is necessary to consider also the question of the compatibility of the cylinder-web diagrams obtained by applying $T$ to 2.1 and 2.2 and, respectively, to 3.11 and 3.12 . For example an application of Lemma 1.5 to the diagram

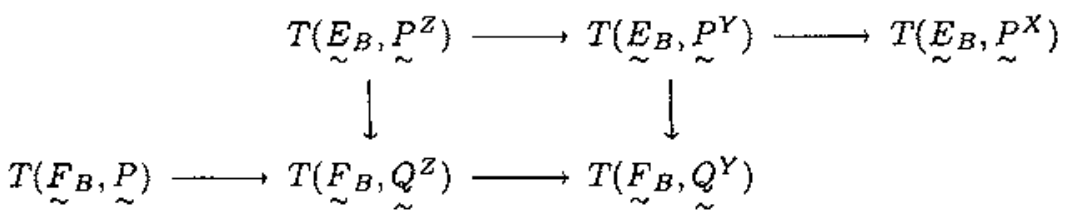

in which the vertical arrows are induced by $\underset{\sim}{E_{B}} \rightarrow \underset{\sim}{F_{B}}$ and $\underset{\sim}{Q^{Z}} \rightarrow \underset{\sim}{P}$, establishes the degree of compatibility of the two versions of the long exact sequence

$$
0 \rightarrow R^{0} T(B, Z) \rightarrow R^{0} T(B, Y) \rightarrow \dot{R}^{0} T(B, X) \rightarrow R^{1} T(B, Z) \rightarrow \ldots
$$


3.17. Remark. If one displays the cylinder web diagram as a doubly infinite array (see for example 0.3 ) then many objects and arrows are duplicated. To avoid unnecessary duplication only three horizontal long sequences need to be written in. The diagram 3.18 displays the full Wall diagram obtained by applying $T$ to the data 0.1 . The long exact sequences are all included but (except for the three horizontal sequences) every third arrow is suppressed. Each pair of arrows $\rightarrow \cdot \rightarrow$ in the same direction is understood to be exact and the sequence continues after three parallel shifts to the right, so that the missing (linking) arrow can be recovered. Although, as discussed above, each diagonal sequence is compatible with the cylinder-web diagram (the horizontal and vertical sequences) it is not the case that each cell of the diagram is commutative or anticommutative. Street [11] has given a description of the identities to be expected in a typical Wall diagram.

\section{Diagram 3.18}

$$
0 \rightarrow R^{0} T(A, Z) \rightarrow R^{0} T(A, Y) \rightarrow R^{0} T(A, X) \rightarrow R^{1} T(A, Z) \rightarrow R^{1} T(A, Y) \rightarrow \ldots
$$

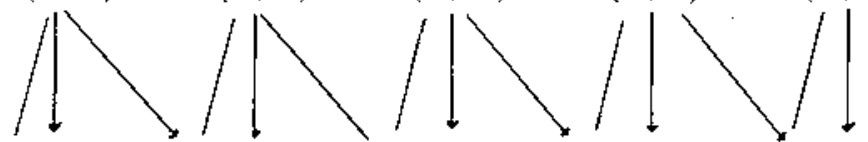

$R^{0} T(B, Z) \rightarrow R^{0} T(B, Y) \rightarrow R^{0} T(B, X) \rightarrow R^{1} T(B, Z) \rightarrow R^{1} T(B, Y) \rightarrow \cdots$

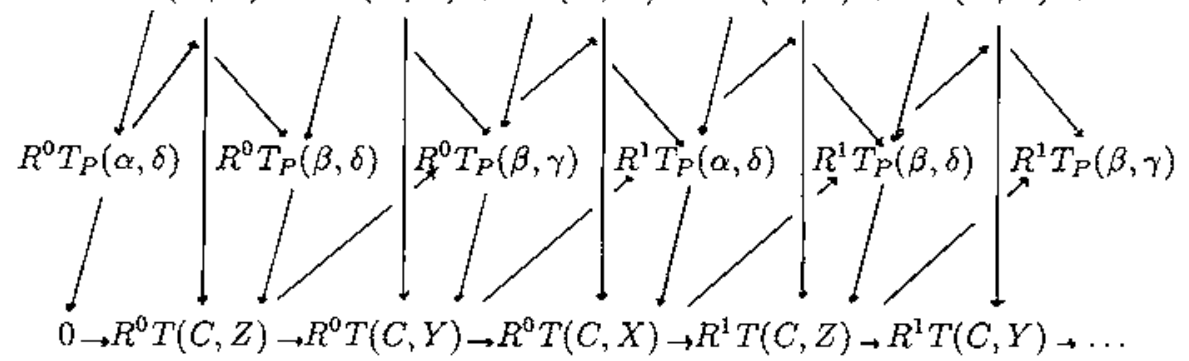

\section{The cases of Hom and $\otimes$}

As mentioned above the theory can be adapted to the case of functors of other variance. The Wall Diagram obtained by applying the bifunctor Hom to the data 0.1 is shown. 
$0 \rightarrow \operatorname{Hom}(Z, A) \rightarrow \operatorname{Hom}(Y, A) \rightarrow \operatorname{Hom}(X, A) \rightarrow \operatorname{Ext}^{1}(Z, A) \rightarrow \operatorname{Ext}^{1}(Y, A) \rightarrow \ldots$

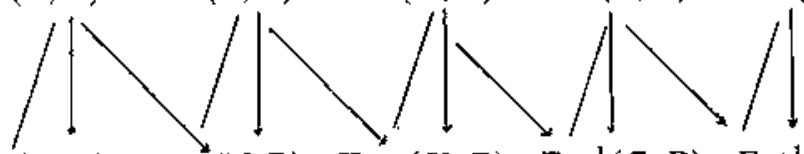

$\operatorname{Hom}(Z, B) \rightarrow \operatorname{Hom}(Y, B) \rightarrow \operatorname{Hom}(X, B) \rightarrow \operatorname{Ext}^{1}(Z, B) \rightarrow \operatorname{Ext}^{1}(Y, B) \rightarrow \cdots$

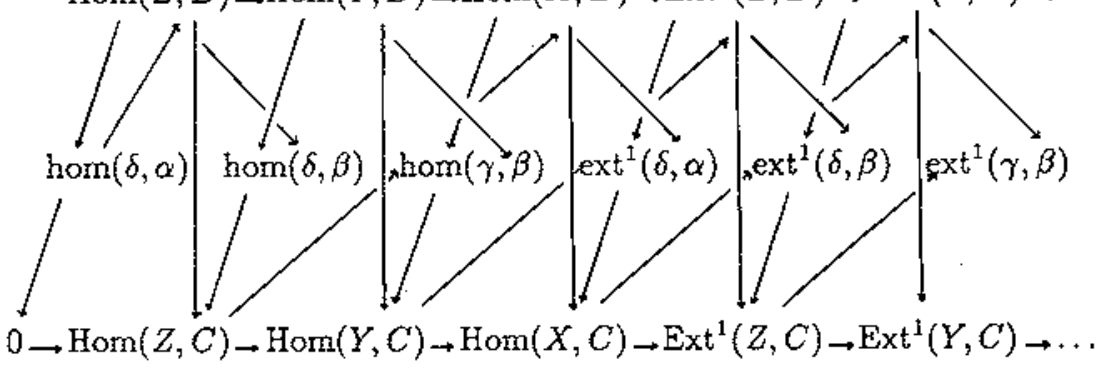

4.2. Remark. As a corollary to Theorem 0.8 (modified form) we recover the natural isomorphisms ext ${ }^{n}(\gamma, \alpha) \approx \operatorname{ext}^{n}(\delta, \beta)$ found in [4, Theorem 2.1].

4.3. Remark. A version of the theory can also be given for left derived functors. For example, let $A$ denote the category of modules over a commutative ring $R$ with unit and $\tilde{l}_{\text {let }} \otimes=$ tens denote the tensor product in $A$. We shall denote the $n$ 'th left derived functor of tens $Q$ by tor $_{n}$. The Wall diagram obtained by applying tens to the pair 0.1 is as indicated:

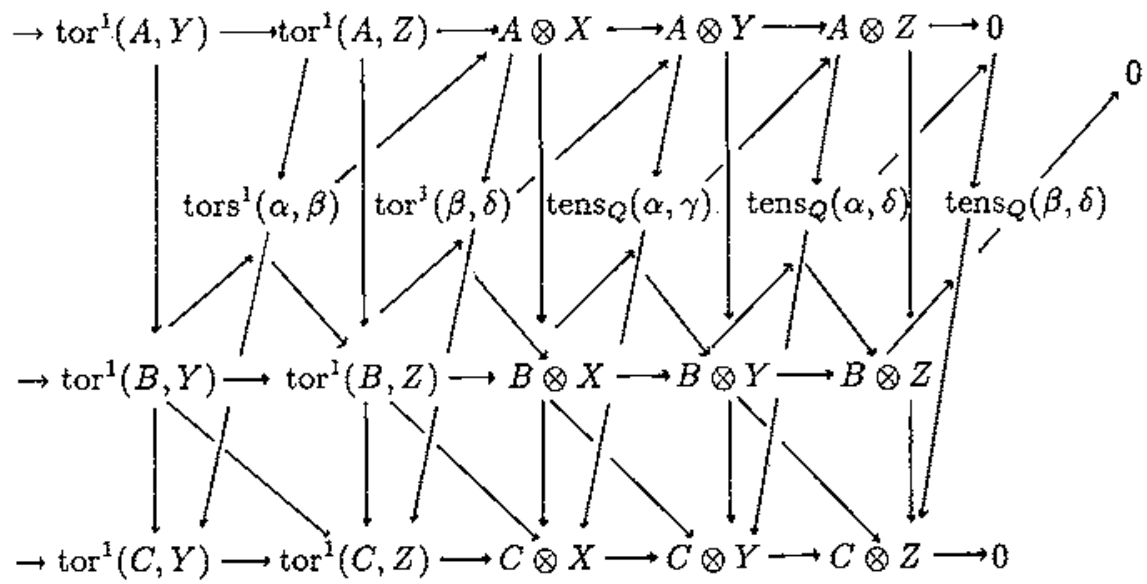

As a corollary of Theorem 0.8 (modified form) we obtain the natural isomorphisms:

$$
\operatorname{tens}_{Q}(\alpha, \delta) \approx \operatorname{tens}_{Q}(\beta, \gamma) ; \operatorname{tor}_{n}(\alpha, \delta) \approx \operatorname{tor}_{n}(\beta, \gamma) \quad(n \geq 1)
$$




\section{Computation of ext $t^{n}$ and tor . $_{n}$.}

The goal of this section will be to compute, in terms of group cohomology and homology, invariants such as $\operatorname{ext}^{n}(\gamma, \beta)$ and $\operatorname{tor}_{n}(\alpha, \delta)$ in certain special cases. Accordingly we consider $A$ to be the category of left $R$-modules where $R=Z G$ is the group ring of a group $G$.

Following standard practice we regard any left $G$-module $M$ as a right $G$ module by setting $m g=g^{-1} m(m \in M, g \in G)$ and in this way interpret the tensor product $M \otimes_{G} N$ of two left $G$-modules. Then $M \otimes_{G} N$ is precisely the quotient of $M \otimes N=M \otimes Z N$ (the tensor product of the underlying abelian groups) by the diagonal action of $G$ on $M \otimes N: g .(m \otimes n)=g m \otimes g n$. Via the pushout diagram

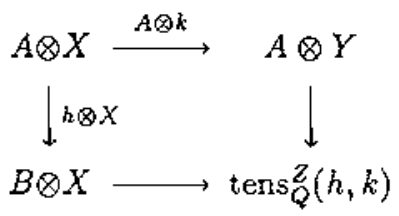

a diagonal action of $G$ on tens ${ }_{Q}^{Z}(h, k)$ is determined and we remark that tens $Q$ $(h, k)=$ tens $_{Q} G(h, k)$ (defined by a pushout corresponding to 5.1 with $\otimes_{G}$ replacing $\otimes)$ is the quotient of tens $Z(h, k)$ by the diagonal action. Similarly for left $G$-modules $M$ and $N, \operatorname{Hom}_{Z}(M, N)$ (the hom set of underlying abelian groups), admits a diagonal action given by

$$
(g u)(m)=g \cdot u\left(g^{-1} m\right) \quad\left(g \in G, u \in \operatorname{Hom}_{Z}(M, N), m \in M\right) .
$$

Since $g u=u$ if and only if $u$ commutes with the action of $G$, we have $\operatorname{Hom}_{G}(M$, $N)=\operatorname{Hom}(M, N)^{G}$, the subgroup of homomorphisms invariant under the diagonal action. For $G$-module homomorphisms $h: A \rightarrow B$ and $k: X \rightarrow Y$ we define hom ${ }_{Z}(h, k)$ via the pullback

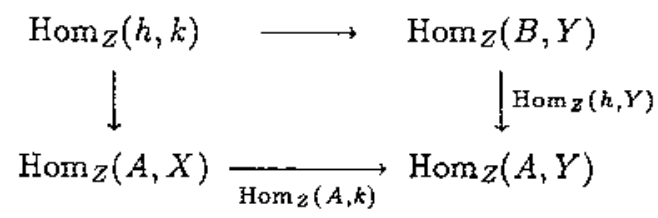

inducing a diagonal action on $\operatorname{hom}_{Z}(h, k)$. Note that $\operatorname{hom}_{G}(h, k)=$ hom $(h, k)=$ hom $g(h, k)^{G}$. We shall require the following lemma.

5.2 Lemma. For a $G$-module $M$ and $G$-module homomorphisms $h: A \rightarrow B$ and $k: X \rightarrow Y$ we have natural isomorphisms

(i) $\operatorname{tens}_{Q}(M \otimes h, k) \approx M \otimes_{G} \operatorname{tens} Z(h, k)$,

(ii) $\operatorname{hom}_{G}(M \otimes h, k) \approx H_{o m_{G}}\left(M, h o m_{Z}(h, k)\right)$, where tens $Q(h, k)$, hom $_{Z}(h, k)$ and the domain and codomain of $M \otimes h$ carry the diagonal action. 
Proof (i): We have a diagram

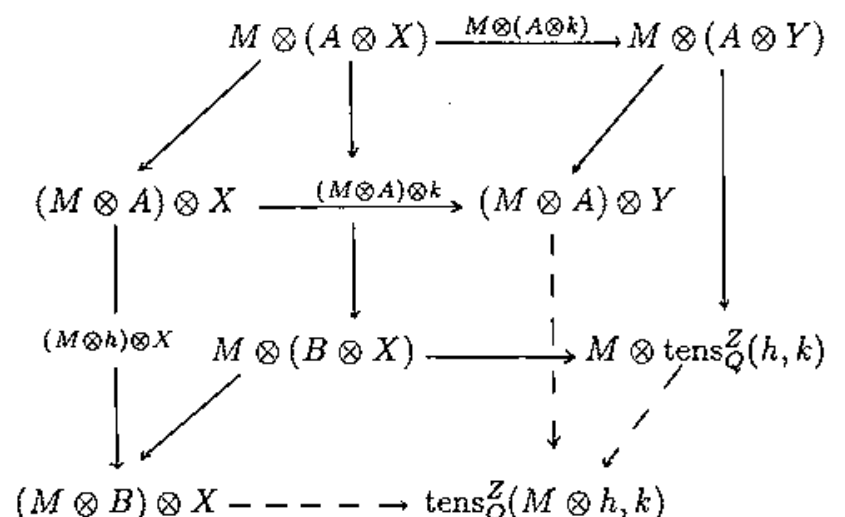

in which the solid slanting arrows are associativity isomorphisms. Since the functor $-\otimes M$ is left adjoint to $\operatorname{Hom}_{Z}(M,-), M \otimes-$ preserves pushouts and hence the dotted slanting arrow is a natural isomorphism. The desired isomorphism is now obtained by factoring out by the diagonal action. The argument for the proof of (ii) is a similar one involving the diagram

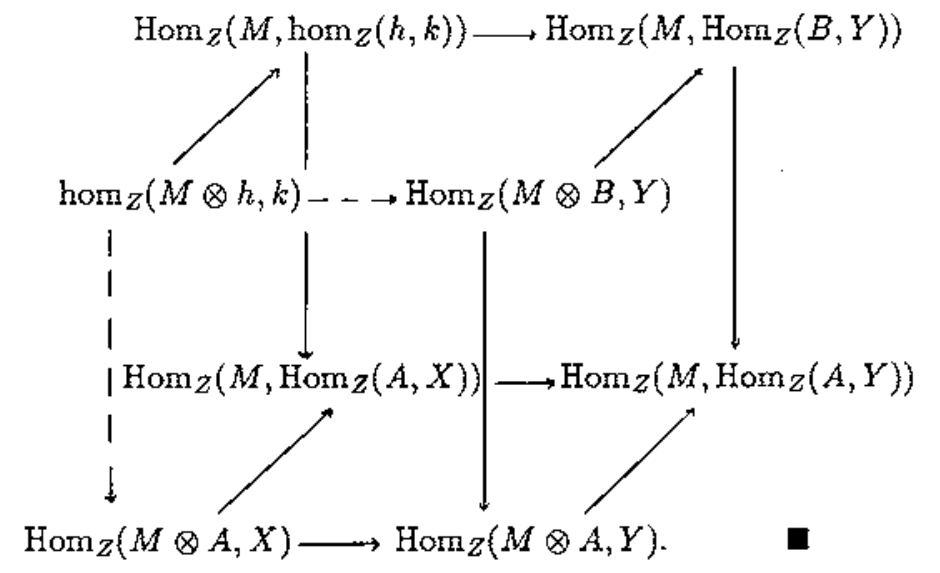

We define tor ${ }_{n}^{G}$ (respectively $\operatorname{ext}_{G}^{n}$ ) to be the $n$-th left derived functor of tens ${ }_{Q}^{G}$ (respectively the $\mathrm{n}$-th right derived functor of hom $\mathrm{m}_{G}$ ). The following result generalizes formulae well-known in the absolute case. [2, Prop. III. 2.2].

5.3 Theorem. Let $\alpha: A \rightarrow B, \gamma: X \rightarrow Y, \delta: Y \rightarrow Z$ be $G$-module homomorphisms.

(i) If $A$ and $B$ are $Z$-torsion free and $\gamma$ is monic then tor $_{*}^{G}(\alpha, \gamma) \approx H_{*}(G$, tens $Z_{Q}(\alpha, \gamma)$ ), where $G$ acts diagonally on tens $Z(\alpha, \gamma)$.

(ii) If $A$ and $B$ are $Z$-free and $\delta$ is epic then ext $t_{G}^{*}(\alpha, \delta) \approx H^{*}\left(G\right.$, hom $_{Z}$ $(\alpha, \delta)$ ) where $G$ acts diagonally on hom $Z(\alpha, \delta)$. 
Proof (i): Let $P^{\gamma} \rightarrow \gamma, P^{\alpha} \rightarrow \alpha$ be projective resolutions. Note that. we can regard $\underset{\sim}{P^{\gamma}}: P_{\sim}^{P} \rightarrow \underset{\sim}{P^{Y}}$ and $\underset{\sim}{P^{\alpha}}: \underset{\sim}{P^{A}} \rightarrow{\underset{\sim}{P}}^{B}$ as chain maps where $P^{X}, P^{Y}, P^{A}, P^{B}$ are projective resolutions of $X, Y, \tilde{A}, B$ respectively. Suppose also that $\tilde{F} \rightarrow Z$ is a projective resolution of the trivial $G$-module $Z$. As discussed in $[2$, pp. 56, 61], $F \otimes A \rightarrow A$ is a flat resolution of $A$. Moreover there is a morphism of resolutions $P^{\alpha} \rightarrow F \otimes \alpha$ which induces a morphism of pushouts

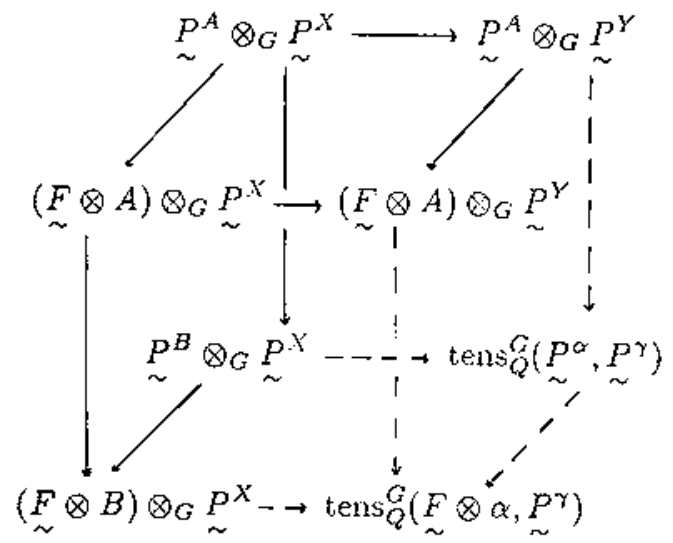

and of short exact sequences of complexes

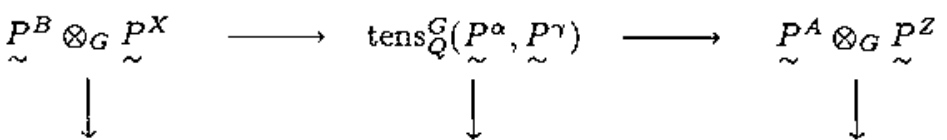

$$
(\underset{\sim}{F} \otimes B) \otimes G \underset{\sim}{P} \longrightarrow \operatorname{tens}_{Q}^{G}\left(\underset{\sim}{F} \otimes \alpha, \underset{\sim}{P^{\gamma}}\right) \longrightarrow\left(\underset{\sim}{F \otimes A} \otimes \otimes_{G} \underset{\sim}{P}\right.
$$

where $Z$ denotes coker $\gamma$ and $\underset{\sim}{P} \rightarrow \underset{\sim}{P}{ }^{Y} \rightarrow \underset{\sim}{P}$ is the associated short exact sequence of resolutions. The outer vertical arrows of 5.4 induce isomorphisms in homology by [2, Theorem I.8.6] and hence, after an application of the five lemma, we have $\operatorname{tor}_{*}^{G}(\alpha, \gamma)=H_{*}\left(\operatorname{tens}_{Q}^{G}\left(P_{\sim}^{\alpha}, P_{\sim}^{\gamma}\right) \approx H_{*}\left(\operatorname{tens}_{Q}^{G}\left(F \otimes \alpha, P_{\sim}^{\gamma}\right)\right.\right.$. A similar argument involving the morphism of resolutions $P^{\gamma} \rightarrow \gamma$ establishes the further isomorphism

$$
H_{*}\left(\operatorname{tens}{ }_{Q}^{G}\left(\underset{\sim}{F} \otimes \alpha, P_{\sim}^{\gamma}\right)\right) \approx H_{*}\left(\operatorname{tens} s_{Q}^{G}(\underset{\sim}{F} \otimes \alpha, \gamma)\right) .
$$

Applying 5.2 (ii) we now have

$$
H_{*}\left(\operatorname{tens}_{Q}^{G}(\underset{\sim}{F} \otimes \alpha, \gamma)\right) \approx H_{*}\left(\underset{\sim}{F} \otimes G \operatorname{tens}_{Q}^{Z}(\alpha, \gamma)\right) \approx H_{*}\left(G, \operatorname{tens}_{Q}^{Z}(\alpha, \gamma)\right),
$$

as required. 
(ii) In this case $\underset{\sim}{F} \otimes A \rightarrow A, \underset{\sim}{F} \otimes B \rightarrow B$ are projective resolutions. Let $X=$ kernel $\delta$ and consider the diagram

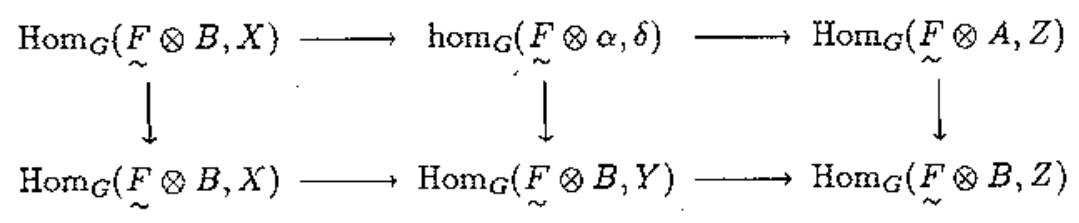

in which the right hand rectangle is a pullback and the rows are short exact sequences of cochain complexes. If $P^{\alpha}$ is a projective resolution of $\alpha$ then there is a morphism of resolutions $\underset{\sim}{P^{\alpha}} \rightarrow \underset{\sim}{F} \otimes \alpha$ inducing a morphism of short exact sequerices

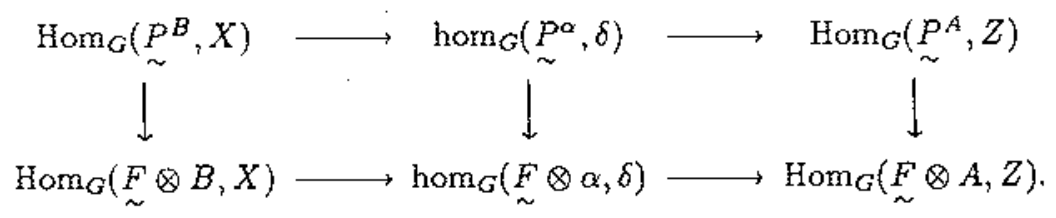

Since the outer vertical arrows induce cohomology isomorphisms we have

$$
\begin{gathered}
\operatorname{ext}_{G}^{*}(\alpha, \delta)=H^{*}\left(\operatorname{hom}_{G}\left(\underset{\sim}{P^{\alpha}}, \delta\right)\right) \approx H^{*}\left(\operatorname{hom}_{G}(\underset{\sim}{F \otimes \alpha, \delta)) \approx}\right. \\
H^{*}\left(\operatorname{hom}_{G}\left(\underset{\sim}{F}, \operatorname{hom}_{Z}(\alpha, \delta)\right)\right)=H^{*}\left(G, \operatorname{hom}_{Z}(\alpha, \delta)\right), \text { as required }
\end{gathered}
$$

5.5. Remark. If the group $G$ is abelian then $M \otimes_{Z G} N$, as normally defined, need not coincide with $M \otimes_{G} N$ as defined above. $\left(M \otimes_{Z G} N\right.$ is obtained from $M \otimes_{Z} N$ by factoring out by the antidiagonal action.) Similar remarks apply to the derived functors $\operatorname{tor}_{n}^{Z G}$ and $\operatorname{ext}_{Z G}^{n}$.

Acknowledgements. The second author acknowledges a grant to the Topology Research Group from the Foundation for Research Development (FRD) of the South African Council for Scientific and Industrial Research.

\section{References}

1. H.B. BRINKMANN AND D. PUPPE, "Abelsche und exakte kategorien, Korrespondzen," Lecture Notes in Math., Springer-Verlag, 96, 1969.

2. K.S. BROWN, "Cohomology of Groups," Springer-Verlag, 1982.

3. H. CARTAN AND S. EILENBerg, Homological Algebra, Princeton University press (1956), Princeton, New Jersey.

4. T.H. FAY AND K.A. HARDIE, Bivariant long exact sequences I, Quaestiones Math. 9 (1986), 207-226. 
5. T.H. FAY, K.A. HARDIE AND P.J. HILTON, The two-square Lemma, Publicacions Matematiques 33, 1 (1989), 133-137, Barcelona.

6. K.A. HARDiE, Bifunctors and the diagonal exact sequences of a cylinder-web diagram, Quaestiones Math. 3 (1979), 167-179.

7. I.S. PRESSMAN, Functors whose domain is a category of morphisms, Acta Math. 118 (1967), 223-249.

8. K.A. HARDIE AND A.V. JANSEN, Toda brackets and the category of homotopy pairs, Quaestiones Moth. 6 (1983), 107-128.

9. K.A. HARDIE AND A.V. JANSEN, Exact sequences in stable homotopy pair theory, Trans. Amer. Math. Soc. 286 (1984), 803-816.

10. I.S. PRESSMAN, The bivariant long exact sequence for the Ext functor, J. Pure and Applied Algebra 6 (1975), 133-153.

11. R. STREET, Homotopy classification by diagrams of interlocking sequences, Math. Colloq. Univ. Cape Town 13 (1984), 83-120.

12. C.T.C. WALL, On the exactness of interlocking sequences, L'Enseign. Math.(2) 12 (1966), 95-100.

Keywords. Derived functors, bivariant long exact sequences, Hom-functor, tensor product, torsion product.

\author{
Temple H. Fay: Department of Mathematics \\ University of Southern Mississippi \\ Hattiesburg, MS 39406-5045 \\ USA \\ Keith A. Hardie: Departrment of Mathematics \\ University of Cape Town \\ Rondebosch 7700 \\ Republic of South Africa
}

Rebut el 25 d'Octubre de 1988 